\title{
Tape Dosage Form
}

National Cancer Institute

\section{Source}

National Cancer Institute. Tape Dosage Form. NCI Thesaurus. Code C47897.

A solid composed of narrow woven fabric or a narrow extruded synthetic material, usually with an adhesive on one or both sides. 